\title{
Primer abordaje antracológico para el valle de Tafí (Tucumán, Argentina)
}

\author{
4 María G. Aguirre*, José L. Peña Monné *** y María M. \\ Sampietro Vattuone*
}

Recibido:

9 de marzo de 2018

Aceptado:

1 de octubre de 2018

\begin{abstract}
Resumen
En este trabajo se presentan los resultados obtenidos al estudiar un conjunto de restos antracológicos recuperados en un perfil estratigráfico ubicado en el valle de Tafí (Tucumán, Argentina). La identificación taxonómica de los macrorrestos se realizó mediante la comparación de los carbones con una colección de referencia de cortes histológicos que incluye ejemplares de la flora del área de estudio. Los carbones se examinaron observando las secciones transversal, longitudinal tangencial y longitudinal radial del leño bajo lupa binocular y microscopio óptico con luz incidente, y se tomaron fotografías con microscopio electrónico de barrido. En el conjunto de carbones se identificaron los siguientes taxa: Baccharis sp., Chuquiraga sp., Alnus sp. y Chusquea sp. Los mismos forman parte de la flora local del área y habrían sido utilizados durante las ocupaciones correspondientes al período de Desarrollos Regionales. Los datos generados alientan al desarrollo de esta línea de trabajo en el área.
\end{abstract}

\section{First anthracological approach for the Tafí valley (Tucumán, Argentina)}

\begin{abstract}
This paper presents the results of the analysis of a set of anthracological remains recovered in a stratigraphic profile located in the Tafi valley (Tucumán, Argentina). We carried out the macro-remains taxonomic identification by comparing the charcoals with a reference collection of histological sections that includes specimens of the study area flora. The charcoals were examined by observing the three diagnostic sections of the stem anatomy with a binocular magnifying glass, an optical microscope with incident light, and photographs were taken with a scanning electron microscope. We identified the following taxa: Baccharis sp., Chuquiraga sp., Alnus sp. and Chusquea sp. They are part of the local flora and would have been used during human occupations corresponding to the Regional Developments period. The data generated in this study encourages the development of this line of work in the area.
\end{abstract}

\footnotetext{
* Instituto Superior de Estudios Sociales (ISES), Facultad de Ciencias Naturales e Instituto Miguel Lillo, Universidad Nacional de Tucumán (UNT) - CONICET. Miguel Lillo 205 (CP T400oJFE), San Miguel de Tucumán, Tucumán, Argentina. E-mail: mgabaguirre@hotmail.com; sampietro@tucbbs.com.ar

** Departamento de Geografía y Ordenación del Territorio, Universidad de Zaragoza. Pedro Cerbuna 12 (CP 50009) Zaragoza, España. E-mail: jlpena@unizar.es
}

\section{Palabras clave}

Antracología Combustibles leñosos Noroeste argentino Desarrollos Regionales

Keywords

Anthracology Woody fuel Northwest Argentina Regional Developments period 


\section{Introducción}

El valle de Tafí (Tucumán, Argentina) corresponde a una cuenca intermontana dentro de las Sierras Pampeanas, su origen es tectónico y presenta reactivaciones neotectónicas cuaternarias (Gutiérrez y Mon, 2004). Tiene dirección Noroeste a Sureste, con el fondo entre los 1.800 y 2.500 m s.n.m., y está limitado por las Cumbres Calchaquíes (4.600 m s.n.m.) y las sierras de Mala Mala (2.800 m s.n.m.) al Este, la sierra de Aconquija (4.600 m s.n.m.) por el Oeste y cerro Ñuñorco (3.300 m s.n.m.) por el Sur. El río Tafí es el principal colector hidrológico de la depresión y discurre con dirección Norte-Sur hasta alcanzar la zona de La Angostura (Figura 1).

La ocupación humana del valle se estima a partir de los 2500 años AP de acuerdo a dataciones radiocarbónicas (Sampietro Vattuone y Vattuone, 2005). Hacia el norte, en la quebrada de Los Corrales, se produjo el hallazgo de enterratorios asociados a material lítico datados en $7420 \pm 25$ años AP (Martínez, Mauri, Mercuri, Caria y Oliszewski, 2013). Esta situación lleva a plantear la posibilidad de presencia humana desde momentos más tempranos a nivel regional.

A pesar de las numerosas investigaciones arqueológicas llevadas a cabo en Tafí, los aspectos referidos a la gestión de las leñas y combustibles no han sido abordados hasta el momento, aun cuando el fuego y su uso a lo largo de la historia de la humanidad constituyen temas de interés para diferentes disciplinas, entre ellas la arqueología (Asouti y Austin, 2005; Jardón Giner, 1998). Al respecto, la conjunción de los estudios arqueológicos y de la xilología ha permitido la identificación anatómico-taxonómica de restos leñosos y de carbón recuperados en diversos contextos arqueológicos. Al mismo tiempo, los aspectos relacionados con la recolección, selección y transformación del material leñoso se abordan a través de la consulta de fuentes etnográficas y etnobotánicas, lo cual contribuye a las interpretaciones arqueológicas ya que permiten distinguir a escala temporal actual o subactual, distintas formas de relación entre los grupos humanos y la vegetación (González Urquijo, Ibáñez Estévez, Moreno García, Peña Chocarro y Zapata Peña, 2005; Joly, March, Marguerie y Yacobaccio, 2009; Picornell Gelabert, 2009).

En este contexto, este trabajo se enmarca en el campo de conocimiento de la antracología, término que proviene de la palabra griega ántraxakos que significa "carbón ardiente" (Euba Rementeria, 2008). El carbón puede ser el resultado de acciones humanas con fines domésticos o industriales y es producido también en la naturaleza como resultado de incendios naturales y de la actividad volcánica (Scott y Damblon, 2010). Al ser este elemento el producto de fenómenos naturales y culturales, distintas disciplinas se han interesado a lo largo del tiempo en el estudio de sus procesos de formación. Dentro de la arqueobotánica, la antracología hace referencia al "estudio e interpretación de los restos leñosos carbonizados provenientes de suelos o de sitios arqueológicos y permite la reconstrucción de la vegetación leñosa local" (Scheel Ybert, 2004, p. 3). Pique i Huerta y Pique i Huerta (1991) expresan que los estudios antracológicos buscan a) reconstruir la paleovegetación (estratos arbóreos y arbustivos) y a través de ella los paleoclimas y b) reflejar la relación de los grupos humanos con su medio ambiente, el aprovechamiento de los recursos forestales y la incidencia antrópica en la evolución de las comunidades vegetales.

En cuanto a la información paleoambiental recabada para el valle, hasta el momento se han detectado acumulaciones de ladera, terraza y fondo de valle generadas fundamentalmente desde inicios del Holoceno. Peña Monné y Sampietro Vattuone (2016) y Sampietro Vattuone y Peña Monné (2016) han demostrado la existencia de una secuencia holocena de procesos agradativos/degradativos que afectaron la formación de laderas-terrazas fluviales-abanicos aluviales formando sistemas acoplados. Esta secuencia está compuesta por cuatro etapas: la más antigua (H1) abarca el Holoceno inferior y medio (ca. 13000-4200 años AP), la siguiente (H2), gran parte del Holoceno 


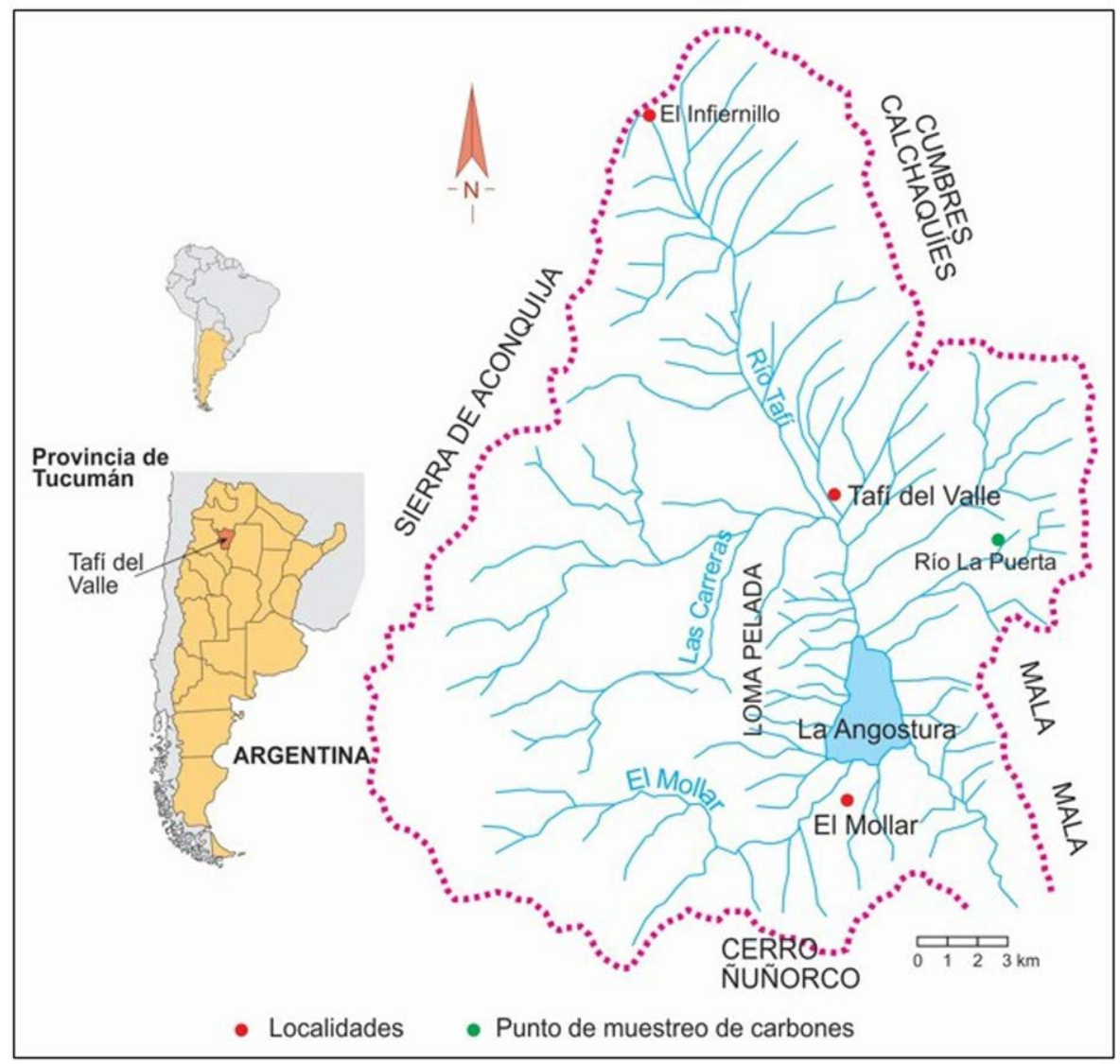

Figura 1. Mapa de situación del área de estudio. Se destaca la ubicación de las principales localidades y el punto de muestro de los carbones.

superior (ca. 4200-600 años AP), y las dos últimas (H3 y H4), fundamentalmente la Pequeña Edad de Hielo y el período reciente, respectivamente.

El objetivo general de este trabajo es identificar taxonómicamente un conjunto de carbones recuperados en un perfil estratigráfico de una ladera acumulativa clasificada como H2s (Sampietro Vattuone y Peña Monné, 2016; Peña Monné y Sampietro Vattuone, en prensa). Esta ladera se formó durante el período comprendido entre $c a .4200$ años AP y poco después de $630 \pm 30$ años AP. El perfil tiene una potencia de $2,5 \mathrm{~m}$ y su secuencia estratigráfica está compuesta por un depósito antiguo, correspondiente al Holoceno inferior y medio, que se extiende desde la base a 2,5 $\mathrm{m}$ de profundidad hasta, aproximadamente, $1 \mathrm{~m}$ de profundidad. Está compuesto por material predominantemente limoso, con un contacto erosivo a techo donde se observa el desarrollo de un canal en el que se concentraron materiales arqueológicos cerámicos, carbones y restos óseos, ubicados entre 0,5 y $1 \mathrm{~m}$ de profundidad. Por las condiciones en que se halla el material y sus características, tema que se desarrollará más adelante, se infiere que la muestra es de depositación primaria o ha sufrido una movilización mínima. La formación de la ladera continuó con posterioridad a la sedimentación de los materiales arqueológicos generando una acumulación de $60 \mathrm{~cm}$ con predominio de arenas y limos grisáceos (Figura 2).

El conjunto de carbones se presentaba asociado a fragmentos de cerámica Famabalasto negro inciso y Santa María bicolor propias del período de Desarrollos Regionales ( $c a$. 1000-1490 A.D.), restos de guano y fragmentos óseos. Una vez individualizados, los carbones de mayor tamaño fueron extraídos del perfil y además se recuperaron muestras de sedimento conteniendo espículas de carbón. Se dejó en el perfil a los restantes 


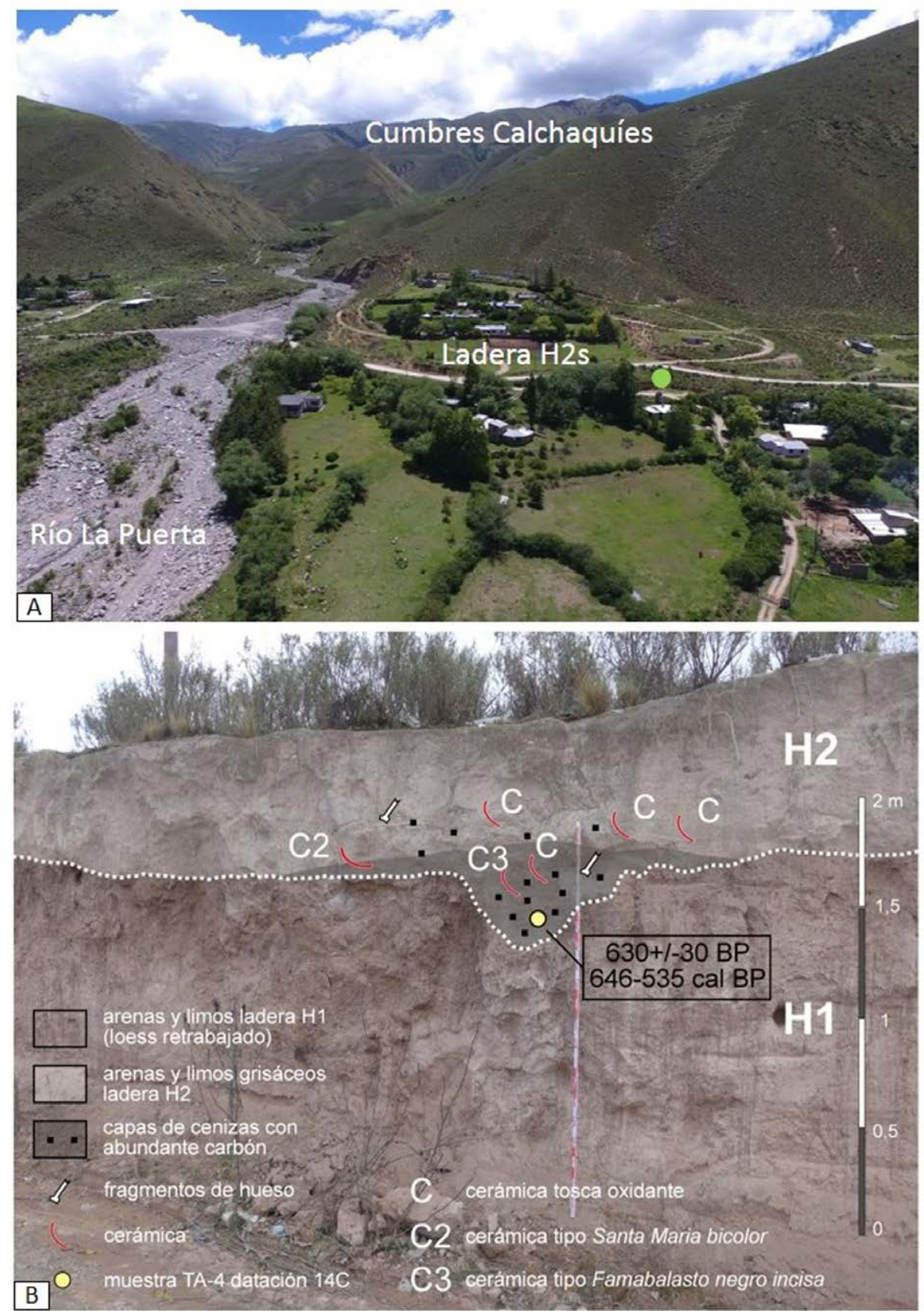

Figura 2. A) Vista general del ápice del cono de La Puerta y el sector de Ladera H2s. El punto de color verde marca la ubicación del perfil de procedencia de los carbones estudiados; B) detalle de la estratigrafía, contenido arqueológico, datación ${ }_{14} C$ y situación de los carbones del perfil analizado.

vestigios arqueológicos. Si bien estos carbones no provienen de un contexto primario de producción, al no contar con antecedentes sobre investigaciones antracológicas para el valle e inclusive para zonas aledañas, consideramos oportuno discutir los resultados taxonómicos obtenidos a modo de reflexiones preliminares.

\section{Área de estudio}

El clima actual del valle es de tipo semiárido, con lluvias concentradas en el verano (87\%) y con fuerte amplitud térmica. La media anual de temperaturas es de $13,1{ }^{\circ} \mathrm{C}$ y la 
de precipitaciones se sitúa en $400 \mathrm{~mm}$ en las áreas bajas, aumentando hacia las laderas montañosas hasta los 500-550 mm. Estos valores están favorecidos por la orientación meridiana del valle, que permite la entrada de vientos húmedos desde el sureste (Sesma, 1987).

Con respecto a la flora, el ascenso desde la llanura tucumana (aproximadamente a $430 \mathrm{~m}$ s.n.m.) hacia Tafí por la vertiente oriental de las Sierras Pampeanas marca un gradiente altitudinal de unos $2.800 \mathrm{~m}$ hasta alcanzar la localidad de El Infiernillo emplazada a $3.100 \mathrm{~m}$ s.n.m. Desde el punto de vista fitogeográfico, durante este trayecto se atraviesan distintos sectores de la provincia biogeográfica de las yungas, el valle en sí se inserta en los pisos superiores de la misma (Pérez Miranda, 2003). A partir de los 1.300 m s.n.m. las especies vegetales del piso basal de la selva dan lugar al desarrollo de elementos arbóreos y arbustivos: palo luz (Prunus tucumanensis Lillo), talilla (Crinodendron tucumanum Lillo), sauco [Sambucus nigras sp. peruviana L. (Kunth.) R. Bolli], Roblina (Ilex argentina Lillo) y aliso (Alnusa cuminata Kunth) (Meyer y Weyrauch, 1966). Esta última especie se presenta formando bosques puros alrededor de los $1.750 \mathrm{~m}$ s.n.m. coexistiendo con algunos saucos y con un sotobosque de salvia blanca [Lepechinia graveolens (Regel) Epling]. A esta altitud, las barrancas abruptas del paisaje son cubiertas por colonias de Chusquea lorentziana (Griseb). El sector del valle conocido como La Angostura (1.990 m s.n.m.) se caracteriza por manchones de achira (Canna compacta Roscoe), Thalictrum decipiens (Boivin) y cortadera (Cortaderia rudiuscula Stapf) (Meyer y Weyrauch, 1966). Al llegar a los 1.850 m s.n.m. termina la vegetación arbórea y se aprecia la región de los prados o pastizales de altura donde predominan Cynodon dactylon (L.) Pers. dactylon, Poa annua L., Sporobolus indicus (L.) R. Br., varias especies de Stipa y pequeñas ciperáceas, mientras que en lugares bajos y anegados se ubican juncáceas de los géneros Luzula DC. y Juncus L. (Meyer y Weyrauch, 1966). Así, el desarrollo de distintas formas de vegetación en el valle varía en función de las condiciones microclimáticas de cada zona y según los distintos sustratos y composiciones del suelo. Puede decirse que Tafí conforma un espacio ecotonal entre la vegetación de las yungas y la del monte y de altura que caracteriza a los valles calchaquíes.

\section{Antecedentes arqueológicos}

Los primeros asentamientos conocidos para el área corresponden a la cultura Tafí (período Formativo, ca. 500 a.C.-1000 A.D.) identificada por sus esculturas líticas (monolitos y máscaras de piedra) (Ambrosetti, 1897) y por un patrón de asentamiento compuesto de estructuras de piedra aisladas o habitaciones circulares alrededor de un patio central, en número variable y en ocasiones formando estructuras agrícolas (González y Núñez Regueiro, 1960). El estudio arqueológico intensivo de sitios como La Bolsa, por ejemplo, señalan que el patrón radial se desarrollaba desde el centro del patio, que además podía contar con estructuras subsuperficiales de planta oval donde se disponían los cuerpos de los difuntos. En los recintos menores adosados se llevaban a cabo actividades de procesamiento de recursos variados (Salazar, Franco Salvi y Berberián, 2011).

El aspecto ceremonial asociado a Tafí corresponde a un montículo vinculado a monolitos de piedra localizado en el sitio arqueológico de Casas Viejas (El Mollar) (González y Núñez Regueiro, 1960). Este sitio constituye, además, un punto importante de conexión entre el valle y la llanura tucumana a través de la quebrada del Portugués. Teniendo en cuenta su cronología, los asentamientos Tafí comenzaron alrededor del 360 a.C. y culminaron hacia 900-1000 A.D. (González y Núñez Regueiro, 1960; Roldán, Maldonado, Urquiza, Vattuone y Sampietro Vattuone, 2016). Se desconocen las causas de la desaparición de esta cultura pero podrían estar relacionadas con un profundo cambio climático hacia condiciones más áridas producido en torno a los 1000 A.D. (Sampietro Vattuone, 2010). 
Más tarde, el valle fue poblado por grupos de la cultura Santa María (período de Desarrollos Regionales, $c a$. 1000-1490 A.D.) procedentes del valle homónimo situado al oeste (Manasse, 2011; Núñez Regueiro y Esparrica, 2010). Siguiendo a Tarragó (2000), las sociedades santamarianas habrían iniciado un proceso expansivo hacia las yungas tucumanas apropiándose de Tafí y estableciendo colonias en demanda de diferentes pisos ecológicos productivos y complementarios. Sus asentamientos son de menor tamaño que los encontrados en el valle de Santa María y se hallan dispersos reutilizando los espacios ocupados durante el periodo Formativo. Se registraron párvulos enterrados en urnas funerarias mientras que la arquitectura residencial fue de diseño cuadrangular con las áreas agrícolas separadas de las residenciales (González y Núñez Regueiro, 1960; Manasse, Orellana y Vaqué, 2009; Núñez Regueiro y Esparrica, 2010). En cuanto a la tecnología cerámica, se han registrado restos de ceramios bicolor y tricolor en unidades domésticas en el sector de Los Cuartos mientras que en La Ovejería se los registró en un cementerio (Páez, Manasse, Ovejero y Toselli, 2005). Las investigaciones llevadas a cabo por Manasse $(2007,2011)$ y Manasse et al. (2009) plantean que la presencia de grupos del tardío en Tafí habría configurado una ocupación humana con dinámica propia, es decir, ocupaciones estables y permanentes en relativa autonomía con respecto a los centros de mayor poder (Manasse, 2007).

Por último, los restos incaicos en el área corresponden al hallazgo de tiestos cerámicos en contextos de excavación y recolección superficial (Páez y Patané Aráoz, 2007) y por estructuras Incas (período Inca ca. 1490-1535 A.D.) escasamente estudiadas y sin dataciones absolutas (Manasse, 2011; Manasse y Páez, 2007). Aún hoy, la forma en que el valle de Tafí responde a las políticas estatales y como se vieron formuladas o reformuladas las relaciones regionales mantenidas en tiempos previos, representan interesantes interrogantes de investigación (Páez y Patané Aráoz, 2007).

Con respecto al manejo de recursos vegetales, Franco Salvi, López y Molar (2014) y Molar (2015) registran micro vestigios de plantas en campos de cultivos y en estructuras asociadas en el sitio arqueológico La Bolsa. Señalan la presencia de silicofitolitos de gramíneas silvestres, Zea mays y cucúrbitas y granos de almidón de semillas afines a Lupinis mutabilis. Por otra parte, Carrizo, Cano y Soler Nixdorff (1999) identificaron macro vestigios de maíz (Zea mays), poroto común o frijol (Phaseolus vulgaris), chañar (Geoffroea decorticans), algarrobo blanco (Prosopis alba), algarrobo negro (Prosopis nigra) y leguminosas mimosoideas en Casas Viejas. A mayor altitud que el valle de Tafí, Carrizo, Oliszewski y Martínez (2003) identificaron la presencia de restos vegetales pertenecientes a Asteraceae, Cucurbitaceae, Fabaceae, Malvaceae, Verbenaceae, Bromeliaceae y Poaceae en el sitio Cueva de Los Corrales. Dichos taxa deben su ubicación en la cueva tanto a actividades antrópicas como a factores naturales.

En cuanto al uso y consumo actual de la flora del área, los trabajos etnobotánicos son escasos pero se disponen de algunos datos que mencionan a Aloysia gratissima (Gill. et Hook.) Tronc., Chenopodium mandonii (Willd.) y Clinopodium gilliesii (Benth.) Kuntze como especies nativas del valle con propiedades terapéuticas (Pastoriza, Martínez Pulido, Budeguer, Nasif y Andrada Mansilla, 2015), mientras que Prunus tucumanensis Lillo (duraznillo del cerro, palo cruz, palo luz) corresponde a una especie peligrosa ya que contiene el glucósido cianogenético lauroceracina (Arambarri et al., 2009).

\section{Método de trabajo}

Una vez individualizada la muestra de carbones en el perfil estratigráfico se procedió a realizar la descripción del mismo y los fragmentos de carbón expuestos se registraron teniendo en cuenta su ubicación estratigráfica y su relación con otros materiales arqueológicos (hueso, cerámica). Una vez fotografiado el conjunto del corte y realizado 
el relevamiento se recuperó cada carbón de manera individual. En laboratorio se confeccionó una histoteca de referencia útil para la identificación taxonómica de los carbones recolectados en campo. Dicha colección incluye a las especies arbustivas y arbóreas locales que se mencionan en la bibliografía sobre la flora del valle (Giusti, Slanis y Aceñolaza 1995; Hyeronimus, 1945; Meyer y Weyrauch, 1966; Vervoorst, 1979). Esta etapa requirió la consulta del herbario de la Fundación Miguel Lillo (LIL), donde se revisaron 13 especies; en esta oportunidad se presentan solo los ejemplares tenidos en cuenta en este trabajo:

》 Baccharis articulata Lam. (Pers.). LIL. 74529. Tucumán. San Pablo. Villa Nougués.

» Chuquiraga calchaquina Cabrera. LIL. 364765. Tucumán. Tafí del Valle. Nuñorco Grande. 2.050 m s.n.m. Leg. F. Versvoost 743.

" Chuquiraga calchaquina Cabrera. LIL. Tucumán. Tafí del Valle. Quebrada de Carapunco, río El Churqui. 2.500 m s.n.m. Col. Schreiter. Det. Frida Gaspar.

Por otra parte, algunos fragmentos de carbón se identificaron a través de la consulta de literatura especializada (Aguirre, Leiton y Becerra, 2006; Tortorelli, 2009).

Con respecto a los aspectos cuantitativos del estudio, se procedió al conteo del número absoluto de restos y a la medición de su peso, mientras que no pudo realizarse el cálculo de la densidad (número de restos por cantidad de sedimento procesado), así como tampoco la estimación de la ubicuidad (porcentaje de las muestras en que cada taxón está presente) ya que los fragmentos se recolectaron como una muestra única y directamente del perfil estratigráfico donde fueron individualizados. Cabe aclarar que los aspectos antes mencionados corresponden a los parámetros comúnmente contemplados en el estudio de conjuntos arqueobotánicos (Banning, 2000; Capparelli, 2009). Además, se efectuó el registro de las características externas de los fragmentos de carbón (erodados, aristas angulosas, etc.) y de cualquier otro rasgo particular que pudieran presentar a fin de obtener indicios sobre los procesos depositacionales y postdepositacionales a los que pudieron estar expuestos.

En cuanto al tamaño de los fragmentos, aspecto relevante en los estudios antracológicos (Chabal, 1992), a fin de obtener una muestra de análisis representativa en este trabajo se optó por trabajar con los carbones que medían $\geq 2 \mathrm{~mm}$. Siguiendo a Pique i Huerta (1999), los caracteres anatómicos de las maderas quemadas son observables independientemente del tamaño del fragmento y a partir de los 2-3 mm ya es posible identificarlos. Teniendo esto en cuenta, la fracción $\leq 2 \mathrm{~mm}$ no fue considerada en este estudio y de la fracción elegida se analizó el 50\%.

El principio sobre el que se basan los estudios antracológicos es la variación interna del leño según cada especie aun cuando el material haya sido expuesto al fuego. En este sentido, la disposición y características de las células que componen el leño permiten la identificación taxonómica del mismo (Castro, 2002; Fahn, 1974; Piqué i Huerta, 1999). Cada carbón arqueológico se fracturó según los planos transversal, longitudinal tangencial y longitudinal radial y las descripciones anatómicas se realizaron siguiendo los criterios cualitativos propuestos por Wheeler, Baas y Gasson (1989) y Aguirre et al. (2006). Los fragmentos se analizaron utilizando microscopio óptico adaptado con iluminación externa a 40x y 100x y su estudio se profundizó empleando el Microscopio Electrónico de Barrido (Supra 55VP) del servicio de microscopia del Centro Integral de Microscopía Electrónica-Consejo Nacional de Investigaciones Científicas y Técnicas (CIME-CONICET). Cabe aclarar que la identificación taxonómica posible de lograrse desde la antracología es variable y en muchas situaciones se llega solamente a nivel de género. 


\section{Resultados}

El total de restos antracológicos recuperados en el perfil estratigráfico fue de 811 fragmentos, de los cuales 387 medían $\leq 2 \mathrm{~mm}$ y 424 tenían un tamaño $\geq$ a $2 \mathrm{~mm}$. De esta última fracción se analizaron 214 fragmentos que constituyen el total de carbones analizados en este trabajo. En general, los carbones eran heterogéneos en cuanto a sus tamaños pero algunos llegaban a medir $2,5 \mathrm{~cm}$. Al observarlos bajo lupa binocular y microscopio óptico se registraron hifas al fragmentarlos. Por otra parte, presentaban superficies erodadas, mientras que en algunos casos los carbones de $\leq 2 \mathrm{~mm}$ tenían formas tabulares, laminares y de aristas angulosas, esta apariencia puede deberse a la desintegración de los fragmentos de mayor tamaño. Al manipular el material se constató que algunos individuos se fracturaban muy fácilmente.

Se identificaron cuatro taxones en la muestra: Baccharis sp. (111 fragmentos), Chuquiraga sp. (89 fragmentos), Alnus sp. (11 fragmentos) y Chusquea sp. (tres fragmentos). La descripción de la anatomía de Baccharis sp. (Figura 3 A-C) y Chuquiraga sp. (Figura $3 \mathrm{D}-\mathrm{F}$ ) se realizó a través del estudio de las tres secciones del leño. En el caso de Alnus sp. (Figura 4A) y de Chusquea sp. (Figura 4 B-C) solo se pudo obtener el corte transversal ya que la fragilidad del material no permitió lograr las demás secciones. En la asignación de los restos de carbón a estos dos géneros se tuvieron en cuenta los caracteres de valor taxonómico observables en la vista transversal según Hoar (1916) para Alnus sp. y Guerreiro, Rodríguez y Rúgolo de Agrasar (2013) para Chusquea sp.

\section{Baccharis sp. Familia Asteracea}

Corte transversal: anillos decrecimiento demarcados por parénquima y vasos en bandas tangenciales. Porosidad semicircular con vasos en patrón diagonal, radial y dendrítico. Algunos vasos solitarios de contorno angular. Fibras de pared delgada a gruesa. Parénquima axial paratraqueal escaso.

Corte longitudinal tangencial: placa de perforación simple, oblicua. Radios de 1-3 células y también de 4-10 células siendo estos predominantes. Punteaduras intervasculares alternas. Fibras septadas.

Corte longitudinal radial: todas las células radiales procumbentes.

\section{Chuquiraga sp. Familia Asteracea}

Corte transversal: anillos de crecimiento indistintos. Porosidad difusa. Predominan los vasos solitarios. Fibras de pared delgada a gruesa. Parénquima axial paratraqueal.

Corte longitudinal tangencial: placa de perforación simple. Punteaduras intervasculares alternas. Engrosamiento helicoidal en vasos. Fibras no septadas. Predominan los radios de 1-3 células y también se observan otros radios multiseriados.

Corte longitudinal radial: células radiales procumbentes y cuadradas.

Los géneros antes descritos comparten rasgos anatómicos por pertenecer a la misma familia botánica (Schweingruber, Börner y Schulze, 2013). De acuerdo a nuestras observaciones, el patrón dendrítico de los vasos en Baccharis y la presencia predominante de vasos solitarios en Chuqiraga podrían ser tomados como rasgos distintivos para diferenciarlos al igual que la predominancia de radios 1-3 seriados en la vista longitudinal tangencial de Chuquiraga. 

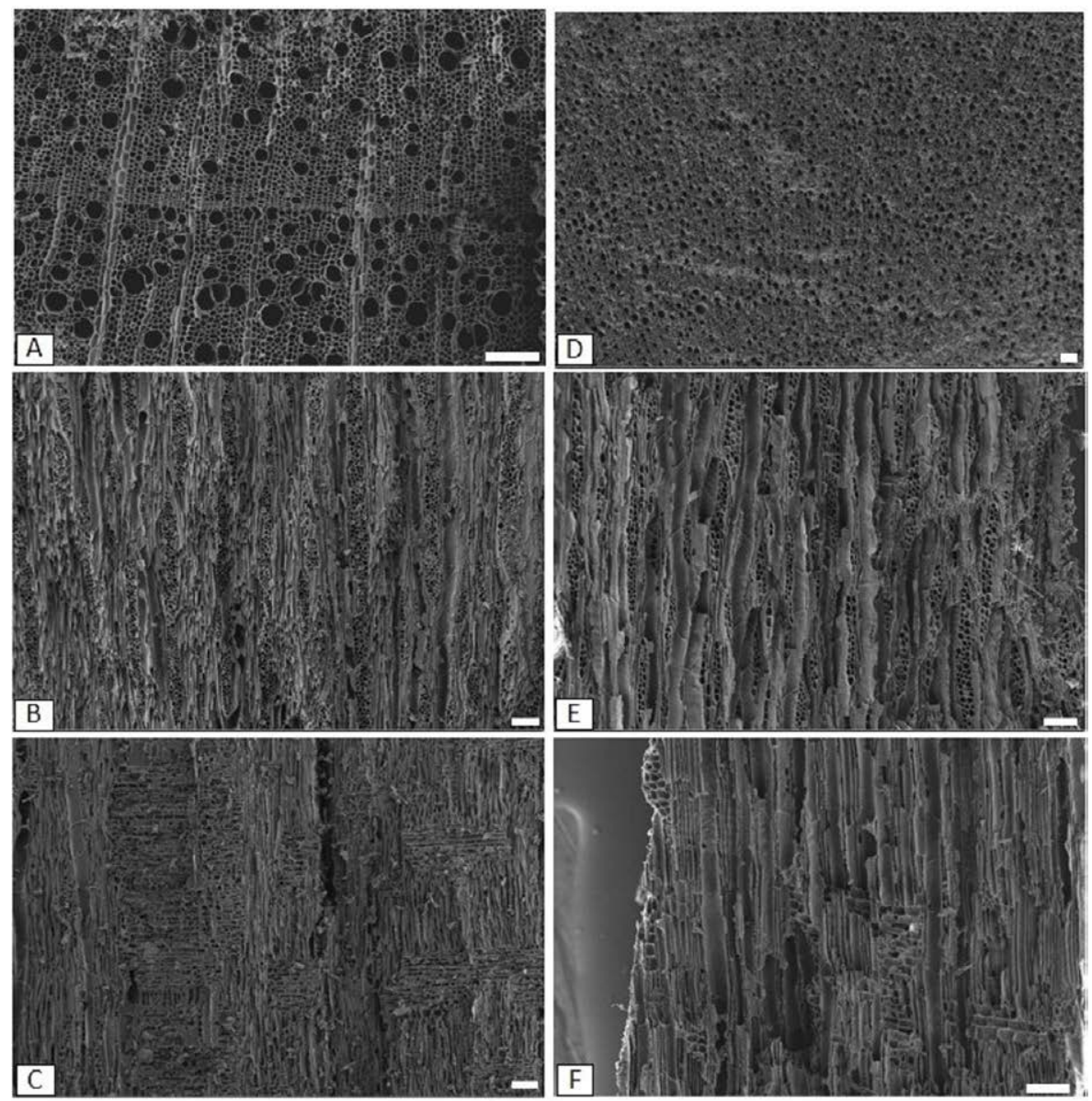

Figura 3. Carbones arqueológicos. Fotografías MEB. A-C) Baccharis sp.: A) corte transversal, 300x; $B$ ) corte longitudinal tangencial, 15ox; C) corte longitudinal radial, 15ox. D-F) Chuquiraga sp.: D) corte transversal, 100x; E) corte longitudinal tangencial, 200x; F) corte longitudinal radial, 250x. Las barras equivalen a $100 \mu \mathrm{m}$.

\section{Alnus sp. Familia Betulaceae}

Corte transversal: los anillos de crecimiento están demarcados por fibro-traqueidas, la porosidad es de tipo semicircular y los vasos se presentan en patrón diagonal y/o patrón radial. Las fibras son de pared delgada. Parenquima axial escaso.

Además de los caracteres observados en este estudio, los géneros que integran esta familia, incluido Alnus, presentan radios formados por una o dos células y raramente por cuatro células, vasos en arreglo radial y radios agregados, siendo excepcionalmente uniseriados en A. acuminata (Hoar, 1916). Tortorelli (2009) observa que en A. acuminata las perforaciones son de tipo escalariformes y los tabiques inclinados. Puntuaciones intervasculares alternas, a veces opuestas con aréola de forma elíptico-oblonga. Los anillos de crecimiento demarcados por una estrecha faja de fibro-traqueidas comprimidas en sentido tangencial. Taquire Arroyo (2016) agrega el registro de poros en cadena de hasta 15 células a lo largo de los anillos de crecimiento.

\section{Chusquea sp. Familia Poaceae}

Corte transversal: no se observaron células epidérmicas. El tallo es macizo y el tejido parenquimático está formado por células circulares y subcirculares de diferentes tamaños. Desde la periferia al centro del tallo, los haces vasculares se distribuyen 

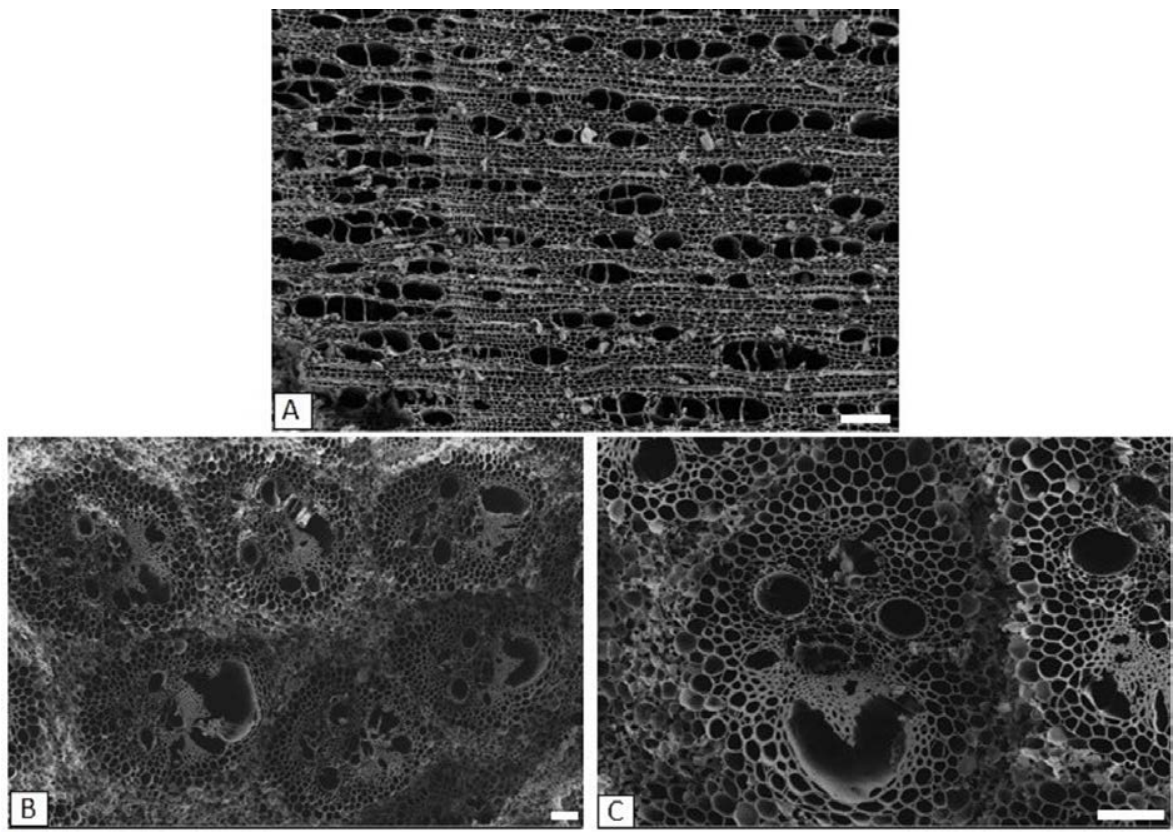

Figura 4. Carbones arqueológicos. Fotografías MEB. A) Alnus sp., corte transversal, 25ox; A-B) Chusquea sp.: B) corte transversal, 150x; C) corte transversal, detalle a $350 x$ de un haz vascular. Las barras equivalen a $100 \mu \mathrm{m}$.

inmersos en el parénquima. Todos los haces están formados por dos vasos de metaxilema, una laguna protoxilemática y el floema que se orienta hacia la cara externa del tallo. Cada haz suele estar separado entre sí por una capa de una a cuatro células de parénquima.

\section{Discusión}

En cuanto a la distribución actual de los taxa registrados, Baccharis L. incluye a especies netamente americanas, siendo algunas de ellas conocidas comúnmente con el nombre de carqueja o carquejilla y utilizadas en el tratamiento de afecciones hepáticas y como diurético (Del Vitto, Petenatti y Petenatti, 1997). En el área de estudio, Giusti et al. (1995) registraron a Baccharis articulata (Lam.) Pers. y a Baccharis tucumanensis Hook. \& Arn. var. tucumanensis. En cuanto al género sudamericano Chuquiraga Juss., en Argentina crecen 16 especies (Zuloaga et al., 1994) desde el sur de Jujuy hasta las sierras de Ambato (Novara, Katinas y Urtubey, 2012). En Tafí habita la especie Chuquiraga calchaquina Cabrera que florece en primavera y crece en las grietas de las rocas donde se acumula agua producida por la condensación del vapor de las nubes que cubren los pastizales entre los 2.300 y los $3.500 \mathrm{~m}$ s.n.m. (Novara et al., 2012). La especie presenta uso ornamental debido a sus vistosos capítulos (Ezcurra, 1985), esto ha generado en el valle una restricción de esta planta hacia los sectores de mayor altitud debido a la presión antrópica orientada a obtener sus flores decorativas. Giusti et al. (1995) registraron a C. calchaquina en los faldeos orientados al sur y sureste del valle de Tafí y en cercanías a la localidad de El Rincón.

Sobre Chusquea sp., Kunth, Nicora y Rúgolo de Agrasar (1987) señalan que este género se distribuye principalmente en las áreas tropicales, subtropicales y templadas del continente americano. En nuestro país se reconocen siete especies (Zuloaga et al., 1994), identificándose en los pisos superiores de las yungas a Chusquea lorentziana y Chusquea deficiens Parodi (Parodi, 1946), ambas asociadas a los bosques de aliso. 
Por último, el género Alnus sp. en la Argentina está representado por cuatro especies, una nativa y tres exóticas que se han escapado de cultivo y naturalizado. En el área de trabajo crece la especie nativa Alnus acuminata Kunth (aliso). Giusti et al. (1995) mostraron que los bosques de aliso no forman una faja altitudinal continua en la región montañosa de Tucumán y advierten una distribución ligada a la disponibilidad de agua, ocupando predominantemente las laderas sur, este y las quebradas sombrías en el valle de Tafí. Dichos bosques alternan con pastizales o bosques de otras especies y los ejemplares actuales que crecen en la zona corresponden a una nueva etapa de crecimiento debido a que ya no están sometidos a la recolección y tala para la obtención de leña (Grau et al., 2007).

Considerando que el objetivo principal de esta investigación fue indagar en la taxonomía del conjunto de carbones recuperados en el perfil estratigráfico, se puede decir que a través de la antracología se registró la presencia de cuatro taxones que forman parte de la flora local de Tafí del Valle (Giusti et al., 1995; Meyer y Weyrauch, 1966; Pérez Miranda, 2003; Zuloaga et al., 1994).

¿Qué significan estos resultados dentro de los temas generales que aborda la antracología? En primer lugar, cuando desde la antracología se busca generar reconstrucciones de paleovegetaciones (Pique i Huerta, 1999; Scheel Ybert, 2004), el registro antracológico debe poseer ciertas características (diversidad taxonómica alta, obtener resultados antracológicos similares entre distintos sitios, concordancia entre resultados generados por disciplinas diversas entre otros; Scheel Ybert, 2004) para elaborar buenas representaciones florísticas. Además, es necesario tener en cuenta que el espectro antracológico está deformado con respecto a la vegetación original ya que las maderas disponibles en el ambiente atraviesan diferentes procesos culturales y naturales (selección, combustión, depositación, etc.) hasta ser recuperadas como carbones en un contexto arqueológico. La información que puede brindarse desde la antracología es un tanto diferente al paisaje actual ya que entre la vegetación pasada y la interpretación paleoecológica existen una serie de filtros (Chabal, 1992). En nuestro caso de estudio, los carbones procedentes del perfil estratigráfico representan una única muestra y los resultados taxonómicos obtenidos no pueden ser comparados con otros de igual naturaleza procedentes del valle. Por el momento, la bibliografía arqueológica no menciona el hallazgo de restos de postes o maderas parcialmente carbonizadas y solo en algunos contextos arqueológicos se han detectado fogones (Salazar et al., 2011; Salazar, Franco Salvi, Berberián y Clavero, 2007; Sampietro Vattuone y Vattuone, 2005) que no cuentan con identificaciones taxonómicas de sus restos. Sitios arqueológicos ya excavados en el valle tampoco han dado cuenta hasta ahora de la presencia de otros órganos (frutos, hojas, etc.) de los taxones registrados desde la antracología. Así, solo puede mencionarse que los carbones de Alnus sp. se suman a los datos polínicos provistos por Garalla, Muruaga y Herbst (2000), quienes plantean que desde los $10350 \pm 80$ años AP hasta los $4120 \pm 60$ años AP hubo una disminución del polen de Alnus sp. (aporte extra-local) hacia la región debido a la debilidad de los vientos del anticiclón del Atlántico. Por todo esto, solo consideramos posible plantear que en este sector de Tafí estaban presentes los cuatro taxones identificados aquí en tiempos prehispánicos.

En cuanto a las investigaciones antracológicas orientadas a reflejar la relación de los grupos humanos con el ambiente, es necesario contemplar en primer lugar, qué tipo de sociedad se está estudiando: cazadores-recolectores, pastores nómades o grupos agricultores, entre otros, ya que cada una de ellas establecerá diferentes formas de interacción con el entorno (Asouti y Austin, 2005). Teniendo esto en cuenta, los pobladores de Tafí durante el período de Desarrollos Regionales responden a una organización social de tipo sedentaria y agrícola-ganadera. Nuestros resultados antracológicos señalan que tres de los cuatro taxa identificados corresponden a restos 
de plantas de hábito arbustivo, siendo Alnus sp. el único taxón de hábito arbóreo registrado en la muestra. Al confrontar estos datos con los obtenidos por Giusti et al. (1995) en censos de vegetación, se desprende una discrepancia debido a que dichos autores contabilizaron al aliso en una frecuencia del $100 \%$ en el área, mientras que especies arbustivas como C. calchaquina o B. articulata se presentaban en el paisaje con un $2 \%$ de frecuencia. Estas diferencias entre investigaciones podrían reflejar la preferencia de los antiguos habitantes del valle hacia ciertas especies leñosas sobre otras durante épocas prehispánicas, teniendo como supuesto de base que los recursos vegetales se mantuvieron estables a lo largo del Holoceno variando solo en cantidad y extensión. Por otra parte, la posible tendencia a utilizar ciertos recursos como combustibles podría asociarse al reconocimiento de propiedades adecuadas de dichos taxones para ser empleados como leña. Se reconoce que la obtención de recursos vegetales, en este caso de leña, se realiza principalmente a través de la recolección, acción que requiere de saberes vinculados al conocimiento de las propiedades de combustibles de las plantas, las áreas de distribución de las mismas, entre otros (Berihuete Azorín y Piqué, 2006). En este sentido, las especies arbustivas producen, en general, leña de fácil encendido que dejan escasos vestigios de combustión, al tiempo que la poda de ramas y la colecta de leña muerta son de relativa facilidad. Al respecto, Ciampagna, Ambrústolo y Zubimendi (2016) señalan que especies de Baccharis sp. de la Patagonia argentina se caracterizan por corresponder a maderas de baja densidad que se emplean como leña iniciadora del fuego. Por otra parte, Andreoni (2015) menciona estudios etnográficos que dan cuenta del empleo del género Chuquiraga sp. también para iniciar el fuego o para realizar señales de humo a distancia. A. acuminata se ubica en el grupo de maderas livianas (Tortorelli, 2009), cuyas propiedades combustibles se relacionan con arder fácilmente pero generando mucha ceniza, mientras que para $C$. lorentziana no se han registrado referencias bibliográficas sobre su uso como combustible. A futuro, la profundización en los estudios antracológicos y la evaluación de otros factores que intervienen en la formación de este tipo de registro (Piqué i Huerta, 1999; Théry-Parisot, Chabalb y Chrzavzeza, 2010) ayudarán a ajustar nuestras inferencias en torno a las estrategias de gestión de recursos forestales (Piqué i Huerta, 1999) operadas en el valle.

Con respecto a las condiciones de hallazgo del conjunto de carbones, la disposición y ubicación de los fragmentos hacen pensar que la dinámica geomorfológica de las laderas de este sector del valle habría ocasionado el arrastre de los restos arqueológicos hacia el sector medio-basal de la geoforma y que en la zona proximal podrían haber existido ocupaciones humanas del período de Desarrollos Regionales, coherentes con las características de los fragmentos cerámicos encontrados que no presentan rodamiento evidenciando poco transporte. Así, la posición final de los restos carbonizados se relacionaría con el transporte natural que sufrieron y se descarta que los restos de carbón deban su formación a la acción de fenómenos de la naturaleza, como por ejemplo un incendio forestal, ya que este tipo de eventos suele observarse macroscópicamente en campo como lentes de carbones asociados a cenizas y a sedimentos de coloraciones diferentes a la matriz de origen. Además, los tamaños de los carbones originados por incendios suelen ser menores a los tamaños que se registraron en este estudio (Lindskoug, 2015; Whitlock, Moreno y Bartlein, 2007). De esta forma, las características del conjunto antracológico estudiado pueden corresponder al tipo denominado por Piqué i Huerta (1999) como acumulaciones de arrastre natural de materiales de combustión, es decir, dispersiones sin una estructura restringida o materiales sueltos sin asociación aparente pero originados según formas particulares de seleccionar, consumir leña y gestionar los residuos del proceso de combustión. Todo esto debe ser comprendido para evitar distorsiones en la interpretación de cualquier registro antracológico (Piqué i Huerta, 1999). Esperamos que, a futuro, estos aspectos puedan ser evaluados en Tafí mediante el estudio de carbones recuperados en contextos claros de ocupación humana. 


\section{Conclusiones}

Los resultados generados durante el estudio antracológico aportan el primer registro de taxones locales empleados como leña para fuego en el área durante el período de Desarrollos Regionales. En sectores carentes de estudios antracológicos, como es el caso del valle de Tafí, el tratamiento de carbones adquiere importancia ya que permite aportar datos acerca de la flora arbustiva y arbórea de espacios donde hubo ocupación humana intensiva durante decenas de años. Al considerar el contexto de procedencia de los restos carbonizados, solo es posible hablar de la carbonización antrópica de los cuatro taxa identificados, mientras que los aspectos relacionados con la gestión del recurso combustible deberán profundizarse a partir de la indagación de contextos primarios de producción del carbón que permitan evaluar la selección, el consumo y el descarte de las leñas. Además, se destaca la necesidad de efectuar análisis de la micromorfología del suelo termoalterado, de rocas y artefactos termoalterados y de cenizas. Por otra parte, los resultados obtenidos no pueden ser aún confrontados con otros de la misma índole ya que los antecedentes disponibles por el momento corresponden a investigaciones efectuadas en sitios arqueológicos emplazados en paisajes diferentes al de Tafí (valle de Hualfín o en el faldeo occidental del Aconquija, por ejemplo) y se obtuvieron en contextos domésticos claramente demarcados. En este sentido, se considera que esta investigación da cuenta de la potencialidad interpretativa preliminar que tienen los restos de carbón y debería animar a la continuidad de los mismos en el valle y en sectores geográficos próximos.

A futuro, se remarca la importancia de incrementar la colección de referencia iniciada y desarrollar, en conjunto con especialistas en etnobotánica, el espectro del uso actual de las plantas del valle, mientras que el trabajo sobre otros registros arqueobotánicos (polen y fitolitos) en curso sobre el perfil permitirán contrastar y complementar los resultados antracológicos aquí presentados.

\section{Agradecimientos}

Los autores de este trabajo agradecen el apoyo brindado por el CONICET a través del Proyecto de Cooperación Internacional R996 profesores invitados, como también al Proyecto MINECO HAR2015-65620-P, al Proyecto PIUNT G520, al personal del Herbario de la Fundación Miguel Lillo y al Servicio de Microscopia CIMECONICET (Tucumán). Parte de este estudio fue realizado en el ámbito del Grupo de Investigaciones Paleoambientales del Cuaternario (PALEOQ) del Gobierno de Aragón y Fondo Europeo de Desarrollo Regional. 


\section{Q Referencias citadas}

» Aguirre, M. G., Leiton, D.yBecerra, M. F. (2006). Techos incendiados y cañas carbonizadas en ámbitos residenciales: análisis antracológico de recursos vegetales provenientes del sitio 2(b) de Campo del Pucará (Catamarca, Argentina). Werken, 9, 5-26.

"Ambrosetti, J. B. (1897). Los monumentos megalíticos del valle de Tafí (Tucumán). Boletín del Instituto Geográfico Argentino, 18, $105-114$.

»Andreoni, D. (2015). Explotación de recursos leñosos en el monte mendocino: el caso del sitio arqueológico Agua de los Caballos-1 (Departamento de San Rafael). Intersecciones en Antropología, 16, 253-269.

» Arambarri, A. M., Freire S., Bayón, N., Colares, M., Monti, C., Novoa, C. y Hernández, M. (2009). Morfoanatomía foliar de árboles medicinales de la Provincia Biogeográfica de las Yungas (Argentina). Boletín Latinoamericano y del Caribe de Plantas Medicinales y Aromáticas, 8(5), 342-379.

»Asouti, E. y Austin, P. (2005). Reconstructing woodland vegetation and its exploitation by past societies based on the analysis and interpretation of archaeological wood charcoal macro-remains. Environmental Archaeology, 10(1), 1-18.

"Banning, E. (2000). The Archaeologist's Laboratory. The Analysis of Archaeological Data. New York: Kluwer Academic Publishers.

» Berihuete Azorín, M. y Piqué, R. (2006). Semillas, frutas, leña, madera: el consumo de plantas entre las sociedades cazadoras-recolectoras. Revista Atlántica Mediterránea de Prehistoria y Arqueología Social, 8, 35-51.

"Capparelli, A. (2009). Intra-site comparison of the archaeoethnobotanical evidence of El Shincal: implicances to the Inka economy. En A. Capparelli, A. Chevalier y R. Pique i Huerta (Eds.), La alimentación en la América precolombina y colonial: un enfoque interdisciplinario. (Treballs d'Etnoarqueologia 7) (pp. 21-35). Madrid: Consejo Superior de Investigaciones Científicas.

»Carrizo, J., Cano, S. y Soler Nixdorff, M. (1999). Recursos vegetales comestibles en el valle de Tafí durante el período formativo: análisis arqueobotánico del sitio Casas Viejas- El Mollar (S TucTav 2). Trabajo presentado en el XII Congreso Nacional de Arqueología Argentina, La Plata, Argentina.

»Carrizo, J., Oliszewski, N. y Martínez, J. (2003). Macrorrestos vegetales del sitio Cueva de Los Corrales (El Infiernillo, Tafí del Valle, Tucumán, Argentina). Revista del Museo Argentino de Ciencias Naturales, 5(2), 253-260.

" Castro, M. A. (2002). Anatomía vegetal y arqueología. En C. Pérez de Micou (Comp.), Plantas y cazadores en Patagonia (pp. 89-103). Buenos Aires: Facultad de Filosofía y Letras, Universidad de Buenos Aires.

" Chabal, L. (1992). La représentativité paléo-écologique des charbons de bois archaéologiques issus du bois de feu. Les Charbons de bois les anciens écosystèmes et le rôle de I'homme. Bulletin de la Société Botanique de France Actualités Botanique, 139, 213-236.

» Ciampagna, M. L., Ambrústolo, P. y Zubimendi, M. A. (2016). Estudios antracológicos en abrigos rocosos de la costa norte de Santa Cruz (Patagonia, Argentina): análisis de los sitios El Oriental y Alero 4. Intersecciones en Antropología, 17, 341-352. 
» Del Vitto, L., Petenatti, E. y Petenatti, M. E. (1997). Recursos herbolarios de San Luis (República Argentina). Primera parte: plantas nativas. Multequina, 6, 49-66.

»Euba Rementeria, I. (2008). Análisis Antracológico de Estructuras Altimontanas en el Valle de la Vansa-sierra del Cadí (Alt Urgell) y en el Valle del Madriu (Andorra): Explotación de Recursos Forestales del Neolítico a Época Moderna. (Tesis Doctoral inédita), Universitat Rovira i Virgili, España.

» Ezcurra, C. (1985). Revisión del género Chuquiraga (Compositae-Mutisieae). Darwiniana, 26(1-4), 219-284.

» Fahn, A. (1974). Anatomía Vegetal. Madrid: H. Blume Ediciones.

»Franco Salvi, V., López, M. L. y Molar, R. (2014). Microrrestos vegetales en campos de cultivo del primer milenio de la Era en el valle de Tafí (Prov. De Tucumán, República Argentina). Arqueología Iberoamericana, 21, 5-22.

» Garralla, S., Muruaga, C. y Herbst, R. (200o). Paleolago El Rincón (Holoceno) Departamento de Tafí del Valle, Provincia de Tucumán, palinología y facies sedimentarias, http://www.unne.edu.ar/unnevieja/Web/cyt/cyt/2000/6_biologicas/b_pdf/b_004.pdf (Acceso: 3 de diciembre, 2015).

» Giusti, L., Slanis, A. y Aceñolaza, P. (1995). Fitosociología de los bosques de Aliso (Alnus acuminata H. B. K. ssp. acuminata) de Tucumán (Argentina). Lilloa, 38(2), 93-120.

» González, A. R. y Núñez Regueiro, V. (1960). Preliminary report on archaeological research in Tafí del Valle, N.W. Argentina. Trabajo presentado en el XXXIV Congreso International de Americanistas. Viena, Austria.

» González Urquijo, J., Ibáñez Estévez, J., Moreno García, M., Peña Chocarro, L. y Zapata Peña, L. (2005). An ethnoarchaeological project in the Eastern Rif (Northern Morocco): first results. Quaderni del Laboratorio di Archeobiologia, 1, 21-32.

» Grau, H., Gasparri, N., Morales, M., Grau, A., Aráoz, E., Carilla J. y Gutiérrez, J. (2007). Regeneración ambiental en el noroeste argentino. Oportunidades para la conservación y restauración de ecosistemas. Ciencia Hoy, 17(100), 46-6o.

» Guerreiro, C., Rodríguez, M. F. y Rúgolo de Agrasar, Z. (2013). Culm anatomy: a contribution to the identification of vegetative Andean woody bamboos in southernmost America. Kew Bulletin, 68(2), 209-218.

» Gutiérrez, A. y Mon, R. (2004). Megageomorfología del valle de Tafí-Aconquija, Tucumán. Revista de la Asociación Geológica Argentina, 59(2), 303-311.

» Hoar, C. (1916). The anatomy and phylogenetic position of the Betulaceae. American Journal of Botany, $3(8), 415-435$.

"Hyeronimus, J. (1945). Observaciones sobre la vegetación de la provincia de Tucumán. Monografías del Instituto de Estudios Geográficos, 5, 1-163.

» Jardón Giner, P. (1998). El ser humano y el control del fuego. En B. Soler Mayor y P. Jardón Giner (Coord.), A la luz del hogar (pp. 14-17). Valencia: Museu de Prehistòria.

» Joly, D., March, R., Marguerie, D. y Yacobaccio, H. (2009). Gestion des combustibles dans la province de Jujuy (Puna, Argentine) depuis l'Holocène ancien: croisement des résultats ethnologiques et anthracologiques. En I. Théry-Parisot, S. Costamagno y A. Henry (Eds.), Proceedings of the gestion des combustibles au Paléolithique et au Mésolithique: Nouveaux outils, nouvelles interprétations (pp. 39-52). Oxford: Archaeopress.

» Lindskoug, H. (2015). Microcarbones: ¿Problema, solución, resolución o sólo otro agujero negro en el estudio de regímenes de fuegos pasados? En C. Belmar y V. Lema (Eds.), Avances y desafíos metodológicos en arqueobotánica: miradas consensuadas y diálogos compartidos desde Sudamérica (pp. 372-378). Santiago: Universidad SEK. 
" Manasse, B. (2007). Tiempo antes de la conquista en el Valle de Tafí. En P. Arenas, B. Manasse y E. Noli (Comp.), Paisajes y procesos sociales en Tafí. Una mirada desde el valle (pp. 141-159). Tucumán: Universidad Nacional de Tucumán.

" Manasse, B. (2011). Arqueología en el Borde Andino del Noroeste Argentino. Sociedades del Último Milenio en el Valle de Tafí, Prov. de Tucumán, República Argentina. (Tesis Doctoral inédita), Universidad Nacional de La Plata, Argentina.

» Manasse, B., Orellana, V. y Vaqué, L. (2009). Contextos arqueológicos superpuestos en Tafí del Valle, Tucumán. En A. Austral y M. Tamagnini (Comps.), Problemática de la arqueología contemporánea (pp. 807-816). Río Cuarto: Universidad Nacional de Río Cuarto.

» Manasse, B. y Páez, C. (2007). El valle de Tafí en tiempos del imperio incaico: inferencias a partir de la tecnología alfarera. Mundo de Antes, 5, 139-156.

» Martínez, J., Mauri, E., Mercuri, C., Caria, M. y Oliszewski, N. (2013). Mid-Holocene human occupations in Tucumán (Northwest Argentina). Quaternary International, 307, 86-95.

» Meyer, T. y Weyrauch, W. (1966). Guía para dos excursiones biológicas en la provincia de Tucumán. Miscelanea, 23, 1-127.

» Molar, R. (2015). Alimentación y reproducción social: biografía de alimentos en contextos aldeanos tempranos del valle de Tafí (2000 AP-100o AP). La Zaranda de Ideas. Revista de Jóvenes Investigadores en Arqueología, 13(1), 41-62.

» Nicora, E. y Rúgolo de Agrasar, Z. (1987). Los géneros de gramíneas de América Austral Argentina, Chile, Uruguay y áreas limítrofes de Bolivia, Paraguay y Brasil. Buenos Aires: Hemisferio Sur.

" Novara, L., Katinas, L. y Urtubey, E. (2012). Asteraceae. Aportes botánicos de Salta-Serie Flora. Salta: Herbario MCNS. Facultad de Ciencias Naturales, Universidad Nacional de Salta.

» Núñez Regueiro, V. y Esparrica, H. (2010). Investigaciones arqueológicas en la zona del km 64,5, Valle de Tafí, provincia de Tucumán. En F. Oliva, N. de Grandis y J. Rodríguez (Comp.), Arqueología argentina en los inicios de un nuevo siglo (pp. 225-237). Rosario: Laborde Libros.

»Páez, M. C., Manasse, B., Ovejero, R. y Toselli, G. (2005). Caracterización tecnológica de alfarería alfarería Santamariana del valle de Tafí. En A. Pifferetti y R. Bolmaro (Ed.), Metodologías científicas aplicadas al estudio de los bienes culturales. Datación, caracterización, prospección, conservación (pp.134-144). Rosario: Humanidades y Artes.

»Páez, M. C. y Patané Aráoz, C. (2007). Análisis tecnológico de alfarería incaica: los platos del Pukara de las Lomas Verdes (Tafí del Valle, Tucumán). Aportes Científicos desde Humanidades, 7, 283-296.

»Parodi, L. (1946). Estudio preliminar sobre el género “Chusquea” en la Argentina. Revista Argentina de Agronomía, 8(4), 331-345.

»Pastoriza, A., Martínez Pulido, L., Budeguer, C., Nasif, A. y Andrada Mansilla, B. (2015). Estudios genéticos en Aloysia gratissima (Gill. et Hook) Tronc., Chenopodium mandonii (S. Watson) Aellen y Clinopodium gilliesii (Benth.) Kuntze. Revista Agronómica del Noroeste Argentino, 35(2), 33-38.

»Peña Monné, J. y Sampietro Vattuone, M. (2016). La secuencia paleoambiental holocena de la vertiente oriental de Loma Pelada (Valle de Tafí, Noroeste Argentino): cambios climáticos y acción humana). En M. Sampietro Vattuone y J. Peña Monné (Eds.), Geoarqueología de los valles Calchaquíes. Ocupaciones humanas y reconstrucciones paleoambientales del Holoceno (pp. 23-64). Tucumán: Universidad Nacional de Tucumán. 
»Peña Monné, J. y Sampietro Vattuone, M. (en prensa). Paleoambientes holocenos del valle de Tafí (Noroeste Argentino) a partir de registros morfosedimentarios y geoarqueológicos. Boletín Geológico y Minero, Numero especial Paleoclimas en Iberoamérica. Un análisis mediante registros geológicos e indicadores ambientales.

»Pérez Miranda, C. (2003). Tucumán y los recursos naturales. Buenos Aires: Secretaria de Agricultura, Ganadería y Pesca de la Nación.

»Picornell Gelabert, L. (2009). Antracología y etnoarqueología. Perspectivas para el estudio de las relaciones entre las sociedades humanas y su entorno. Complutum, 20(1), 133-151.

»Piqué i Huerta, R. (1999) Producción y uso de combustible vegetal arqueológico: Una evaluación arqueológica (Treballs d’Etnoarqueología 3). Madrid: Universidad Autónoma de Barcelona; Consejo Superior de Investigaciones Científicas.

»Piqué i Huerta, R. y Piqué i Huerta, J. (1991). Aplicación del tratamiento de imágenes digitalizadas al análisis antracológico: un ensayo de determinación automática. En A. Vilá (Coord.), Arqueología: nuevas tendencias (pp. 115-129). Madrid: Consejo Superior de Investigaciones Científicas.

» Roldán, J., Maldonado, M., Urquiza, S., Vattuone, M. y Sampietro Vattuone, M. (2016). Suelos antrópicos vs. naturales: La Costa 2 (Valle de Tafí, Tucumán). Arqueología, 22(1), 127-148.

»Salazar, J., Franco Salvi, V. y Berberián, E. (2011). Una aproximación a la sacralidad de los espacios domésticos del primer milenio en Valle de Tafí (Noroeste Argentino). Revista Española de Antropología Americana, 41(1), 9-26.

»Salazar, J., Franco Salvi, V., Berberián, E. y Clavero, S. (2007). Contextos domésticos del valle de Tafí. Werken, 10, 25-47.

"Sampietro Vattuone, M. (2010). Espacio, ambiente y los inicios de la agricultura indígena en el noroeste argentino: Un enfoque geoarqueológico. Madrid: JAS Arqueología.

»Sampietro Vattuone, M. y Peña Monné, J. (2016). Geomorphological dynamic changes during the Holocene through ephemeral stream analyses from Northwest Argentina. Catena, 147, 663-677.

»Sampietro Vattuone, M. y Vattuone, M. (2005). Reconstruction of activity areas at a Formative household in Northwest of Argentina. Geoarchaeology: An International Journal, 20(4), 337-354.

»Scheel-Ybert, R. (2004). Teoria e método em antracologia. Considerações teóricas e perspectivas. Arquivos do Museu Nacional Rio de Janeiro, 62(1), 3-14.

»Schweingruber, F., Börner, A. y Schulze, E. (2013). Atlas of Stem Anatomy in Herbs, Shrubs and Trees (Volumen 2). Berlin: Springer.

»Scott, A. y Damblon, F. (2010). Charcoal: taphonomy and significance in geology, botany and archaeology. Palaeogeography, Palaeoclimatology, Palaeoecology, 291(1-2), 1-10.

»Sesma, J. (1987). Geología del Cuaternario y geomorfología aplicada en el Valle de Tafí. (Tesis de Licenciatura inédita), Universidad Nacional de Tucumán, Argentina.

» Taquire Arroyo, A. (2016) Potencialidad dendroclimática de Alnus acuminata H.B.K. para el Valle del Mantaro. (Tesis Doctoral inédita), Universidad Nacional del Centro del Perú, Perú.

» Tarragó, M. (2000). Chacras y Pukara. Desarrollos sociales tardíos. En M. Tarragó (Ed.), Los pueblos originarios y la conquista. Nueva Historia Argentina (pp.257-300). Buenos Aires: Sudamericana. 
" Théry-Parisot, I., Chabalb, L. y Chrzavzeza, J. (2010). Anthracology and taphonomy, from wood gathering to charcoal analysis. A review of the taphonomic processes modifying charcoal assemblages, in archaeological contexts. Palaeogeography, Palaeoclimatology, Palaeoecology, 291(1-2), 142-153.

» Tortorelli, L. (2009). Maderas y Bosques Argentinos (2ㅌdición). Buenos Aires: Orientación Gráfica.

» Vervoorst, F. (1979). La Vegetación del Noroeste Argentino y su Degradación. Tucumán: Ministerio de Cultura y Educación de la Nación, Fundación Miguel Lillo.

» Wheeler, E., Baas, P. y Gasson, P. (Eds.). (1989). List of Microscopic Features for Hardwood Identification. Leiden: International Association of Wood Anatomists.

» Whitlock, C., Moreno, P. y Bartlein, P. (2007). Climatic controls of Holocene fire patterns in southern South America. Quaternary Research, 68(1), 28-36.

»Zuloaga, F., Nicora, E., Rúgolo de Agrasar, Z., Morrone, O., Pensiero, J. y Cialdella, A. M. (1994). Catálogo de la Familia Poaceae en la República Argentina. Missouri: Missouri Botanical Garden. 